\title{
Comparison of Three retrieval accuracy of image retrieval technology
}

\author{
Bai Qing $\mathrm{HE}^{1, \mathrm{a}}$, Zi Min WANG ${ }^{2}$ \\ ${ }^{1}$ lecturer, master, research direction: information processing, telecommunications management, unit: Nanchang Institute of \\ Technology. \\ ${ }^{2}$ lecturer, master, research direction: information processing, ideological and political management, unit: Nanchang Institute of \\ Technology.
}

\begin{abstract}
With the development of society, it began between the various sectors and industries increasingly high integration of information, and the image retrieval technology has played an increasingly important role in the current exchange of information. Image retrieval faster, higher accuracy, then the computer used in image retrieval technology is more advanced. By now several popular image retrieval technology introduction, pointed out that the principles of these types of image retrieval technology and difficulties, comparative study of the advantages and disadvantages of these three image retrieval technology, so that we can retrieve the image retrieval accuracy further understanding.
\end{abstract}

\section{Introduction}

In the field of visual information research, It has been greatly developed. International Organization for Standardization has developed the MPEG-7 GB [1] for this area, so that relevant research on an international scale with standardized criteria can exercise. With standardization in this field can make international researchers or better commonality between users, easy to exchange and research.

In the field of traditional visual information research for image retrieval is image-based text annotations. That is, when uploading pictures or video information, pictures or videos will be editorial comment, and then the user needs to retrieve text pictures or videos. This method is relatively simple, but there are difficult to solve several problems. First, you must carry out all the pictures or video text notes that in modern society is not very realistic, hundreds of thousands of pictures or videos are uploaded every day, it is unable to complete the task only rely on manpower to text notes. Secondly,Manual text annotation is very subjective.For the same picture or video, different people have different interpretations. This traditional method is only suitable for specific occasions particular search, it is not suitable for all occasions, the search accuracy is relatively low.Comparison and analysis of existing image retrieval technology, we can find that a lot of new search technology has very good advantages, these technologies are more efficient, accurate, and quickly retrieve what we're looking for. Here we briefly describe some of the practical application of the more mainstream image retrieval, retrieval accuracy comparative study between them.

\section{2 color-based image retrieval}

There are two main color-based image retrieval technology. The first technique is based on a picture of the main colors, it is the user through the palette depicted image colors to achieve image retrieval, such as a large blue sky picture main colors can be blue. The second technique is based on the legend, according to which electronic artwork provided by the user, characterized by color pattern analysis computer image retrieval system, and then retrieve the color characteristics similar images in the database. Wherein based on the legend of the technology is not suitable for ordinary users. The main colors in [2] based retrieval method can only describe a color picture information generally has a relatively large error. For example, the user is mainly blue, the computer may retrieve the blue sky or the sea. According to the actual situation of the picture, you can choose two different main colors. Than the picture depicts the desert under the blue sky, you can choose blue and yellow as a picture retrieved two main colors. So you can more accurately retrieve the user desired image.

The first main colors query mode is more commonly used. However, the user selects the main colors when it is based on the naked eye to judge, with the user's subjective color, all of this often with a certain randomness and fuzziness. So when the user selects a main color, it also determines a value, for example, select the red $(R=255, G=0, B=0)$ on the palette, then retrieve the image, the computer only searches for a red that has the value. But this color is only the user through the naked eye to determine the modulation of the main colors, with the real needs of the color still exist certain differences.In this case, the user may have missed out on

\footnotetext{
a Corresponding author: 86035262@qq.com、308809980@qq.com
} 
truly meet the requirements of image information in image retrieval process.

In fact, the range of red included is relatively broad, because the user's randomness and fuzziness, which requires a master tones expansion process. That is, when the user selects the red $(R=255, G=0, B=0)$, the computer system will take this color as the center, expanding the range of RGB, retrieve some other red within a certain range. So that you can visually identify colors and better linked to the theoretical value. So we built a HSV color retrieval model [2].

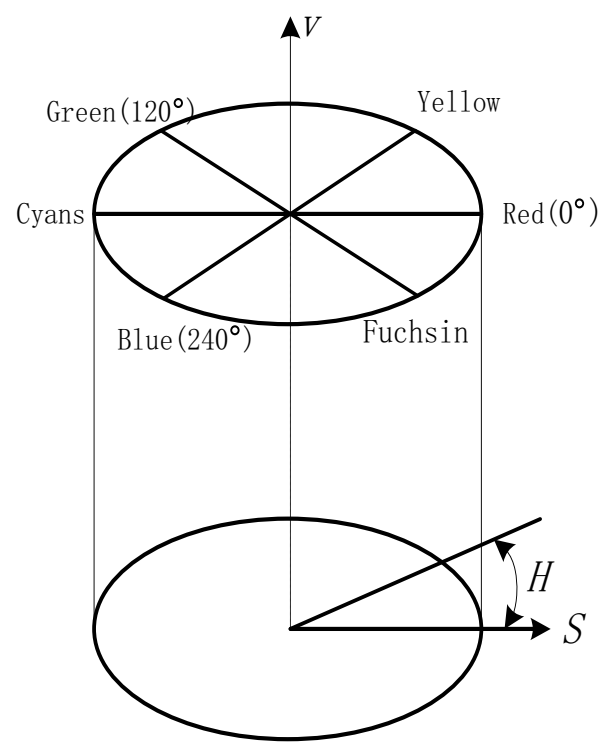

Figure 1 HSV color space schematic

HSV model refers to the color hue, saturation and brightness of the 3 properties, according to the 3 attributes to build a three-dimensional coordinate axes, where $\mathrm{H}$ is by color to identify the colors; saturation $\mathrm{S}$ is showing the depth of color, because also blue, light blue and dark blue also divided; the final $\mathrm{V}$ is light or dark. HSV values to obtain RGB values, the conversion process is as follows:Given the values of RGB color space $(r, g, b), r, g, b \in[0,1, \cdots, 255]$, the conversion to HSV space, $h, s$, v value is calculated as follows:

$\operatorname{suppose} v^{\prime}=\max (r, g, \mathrm{~b}), \mathrm{r}^{\prime}, \mathrm{g}^{\prime}, \mathrm{b}^{\prime}$ is defined as :

$r^{\prime}=\frac{v^{\prime}-r}{v^{\prime}-\min (r, g, b)}, g^{\prime}=\frac{v^{\prime}-g}{v^{\prime}-\min (r, g, b)}, b^{\prime}=\frac{v^{\prime}-b}{v^{\prime}-\min (r, g, b)}$

$v=v^{\prime} / 255$

$s=\frac{v^{\prime}-\min (r, g, b)}{v^{\prime}}$ $h^{\prime}=\left\{\begin{array}{l}\left(5+b^{\prime}\right), r=\max (r, g, b) \& g=\min (r, g, b) \\ \left(1-g^{\prime}\right), r=\max (r, g, b) \& g \neq \min (r, g, b) \\ \left(1+r^{\prime}\right), g=\max (r, g, b) \& b=\min (r, g, b) \\ \left(3-b^{\prime}\right), g=\max (r, g, b) \& b \neq \min (r, g, b) \\ \left(3+g^{\prime}\right), b=\max (r, g, b) \& r=\min (r, g, b) \\ \left(5-r^{\prime}\right), \text { other }\end{array}\right.$

$h=60 \times h^{\prime}$

Here, $r, g, b \in[0 \Lambda 255], h \in[0 \Lambda 360], s \in[0 \Lambda 1]$, $v \in[0 \Lambda 1]$.

The HSV model we used that may represent a very large number of colors, if the histogram vector calculation, will be a complex process. In this case, if the first space proper quantization, then the histogram, will save many. HSV model Bar $\mathrm{H}$ space is divided into 8 parts, saturation is divided into 3 parts, the brightness is also divided into three points, then:

$$
H=\left\{\begin{array}{l}
0 \text { if } h \in[316,20] \\
1 \text { if } h \in[21,40] \\
2 \text { if } h \in[41,75] \\
3 \text { if } h \in[76,155] \\
4 \text { if } h \in[156,190] \\
5 \text { if } h \in[191,270] \\
6 \text { if } h \in[271,295] \\
7 \text { if } h \in[2961,315]
\end{array} \quad s=\left\{\begin{array}{l}
0 \text { if } \mathrm{s} \in[0,0,2] \\
1 \text { if } \mathrm{s} \in[0,2,0,7] \\
2 \text { if } \mathrm{s} \in[0,7,1]
\end{array}\right.\right.
$$

According to the above calculation, it can be thought of feature vectors:

$$
1=\mathrm{H} Q \mathrm{~s} Q \mathrm{v}+\mathrm{SO} \mathrm{v}+\mathrm{V}(1)
$$

Wherein, Q s and Q v S and V components are the quantization levels, taking $\mathrm{Q} s=3, \mathrm{Q} v=3$. Thus (1) can be expressed as:

$$
1=9 \mathrm{H}+3 \mathrm{~S}+\mathrm{V}(2)
$$

Thus, H, S, V these components form a threedimensional vector, and 1 know the range of $[0,1, \cdots, 71]$, the calculation to obtain a one-dimensional histogram 72 1 handle (bin) of [4].

A hypothetical example of the image $\mathrm{Q}$, which is a histogram of feature vectors $\mathrm{H} \mathrm{q}(\mathrm{q} 0, \mathrm{q} 1, \cdots, \mathrm{q} \mathrm{L})$, an image in the image database is $\mathrm{S}$, which is a histogram of 
feature vectors $\mathrm{H} \mathrm{s}(\mathrm{s} 0, \mathrm{~s} 1, \cdots, \mathrm{s} \mathrm{L})$, wherein, $0 \leqslant \mathrm{qi} \leqslant 1$, $0 \leqslant$ si $\leqslant 1$, a normalized scale value, $\mathrm{L}$ is the dimension of a one-dimensional histogram of the vector. then the histogram intersection algorithm [3] image similarity measure, the intersection of two histograms is defined as follows: According to HSV model it is more significant to distinguish between two different images. Color-based image retrieval technology with a certain degree of accuracy.

\section{3 knowledge-based image retrieval}

Knowledge-based image retrieval technology is based on artificial intelligence technology, through understanding and expression of the image, and image database of prebuilt docking, the user needs to retrieve the comprehensive application of technology picture information. Premise of this technique is that the system must be able to recognize natural language and understand the meaning of the language, which can make a judgment.

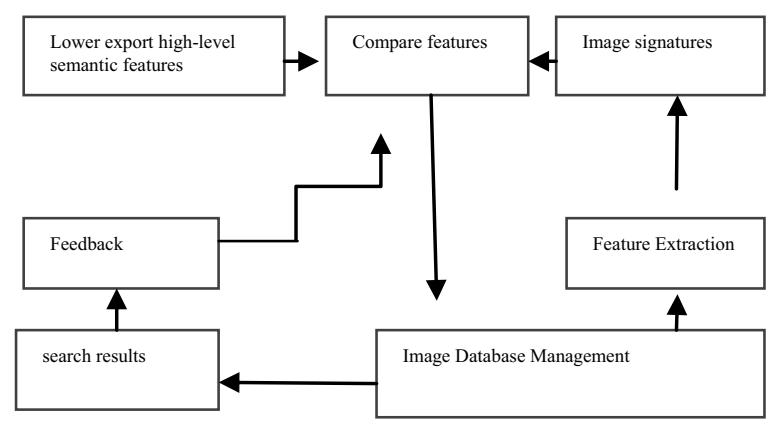

Figure 2 frames

Natural language [5] is the user input language query system. As we all know, natural language semantic structure and changeable, changeable, has the synonym. Let the system recognizes natural language, We need to build intelligent systems, and related language rules and syntax analysis introduced into the system to ensure the retrieval system is in understanding the basis of semantic retrieval, rather than simply matching text. Construction of the intelligent system, which we call the concept of Semantic Network, is highly portable and can be modified, to achieve user requirements for the system to accurately understand and implement the corresponding retrieval.

The construction of intelligent system needs the help of relevant experts. Development Engineer is responsible for building intelligent systems framework itself, but to be embedded in the language rules, we need to consult experts in related fields. First of all, experts in the field need to be grouped into specialized language knowledge of language rules, developed by the engineer in an appropriate form implant system to achieve a computer can preinstalled language database, retrieve the contents of the user to make appropriate judgments and simple reasoning, retrieve the information that users need.
Knowledge-based image retrieval technology in the actual production also has a wide range of applications, it also has a very important feature is the high artificial intelligence [6]. Retrieval system not only can be used to identify the natural language rules, but also through their own learning, the image retrieval process to retrieve the information stored in the database. Thus, in the process of image retrieval system, the system will be self-taught and self-storage mode, ready to improve their recognition rate. From this we can see that the core issue of knowledgebased retrieval technique is to establish contains a wealth of knowledge of the language database.

For now, the knowledge database with the subjectsidedness.For example, knowledge of modern information includes medical, electronic communications, mathematics, history and other aspects. In terms of the form of pictures stored to correspond to a very rich language information, the system has not been able to achieve in all areas of information for accurate identification and retrieval. To solve this problem, we need to work together various experts in the field.

Knowledge-based image retrieval technology to better understand the needs of users, in order to find a more accurate picture of the user needs to retrieve information. Therefore, this technique has higher retrieval accuracy, integrated knowledge-based image retrieval system should have the following characteristics:

1> The system is capable of doing the image NOTE: retrieval system according to the characteristics of the original image analysis knowledge base to derive the relevant semantic description, and then summed up with experts in the field of rule combine to form a new knowledge base.

2> The system is not only able to identify and retrieve relevant semantic input, but also according to semantics, for simple judgment and decision-making of the work content. This fully embodies the intelligent system.

$3>$ system for the average user, it should have a simple user interface, which means that ordinary customers to retrieve the contents of the input, the system can make the search process according to the requirements, without the need for ordinary users with professional knowledge.

\section{4 based on SVM relevance feedback image retrieval algorithm retrieval technology}

First to introduce the principle of SVM [7], SVM principle refers to the object projected onto the highdimensional space or build an optimal classification surface in the original space, so that two different samples can be distinguished separately. Constructed this 
classification is based on two different surface samples furthest from the hyperplane.

SVM principle need points note:

Assuming for the current set of samples (x i, y i) $1 \leqslant \mathrm{i} \leqslant \mathrm{N}$, $\mathrm{x} i \in \mathrm{R}$ d,yi as the class symbols, ranging from $(-1,1)$, linear functional form d-dimensional space corresponding to $\mathrm{g}(\mathrm{x})=\mathrm{w} \cdot \mathrm{x}+\mathrm{b}$, and the corresponding function equation classification plane is $\mathrm{w} \cdot \mathrm{x}+\mathrm{b}=0$. The $\mathrm{g}(\mathrm{x})$ is normalized to give $x i$ are $g(x) \geqslant 1$ solution, that is, the sample surface is a function of classification $\mathrm{g}(\mathrm{x})=1$, can be obtained classified operation interval $2 /\|\mathrm{w}\|$. To solve the optimal classification surface is equivalent to minimize $\|\mathrm{w}\|$, the objective function is:

Using Lagrange multiplier method introduced multiplier $\mathrm{A}=(\mathrm{A} 1, \cdots, \mathrm{AN})$, can be obtained $\mathrm{w}=\Sigma \mathrm{iA}$ iyixi, $x i$ is located spaced sample classification surface, this sample is called support vector [8], classification function:Of course, there is a situation when, this time on the need to introduce slack variables and the penalty factor, the objective function becomes:In the non-linear operation, SVM is also possible to convert the input space to a high-dimensional space, so that you can calculate solved in the new space. Which can become linear:Therefore, SVM FOUR built on the basis of the above statistics, in the actual application process also achieved good results.

Let's look at how to SVM algorithm associated negative feedback retrieval technologies. Relevance feedback process can be seen as two SVM classification model, and a specific search algorithm works as follows:

The first step: You can use the previous method for the user to retrieve a desired image.

Step two: user former N RT [9] images are labeled, can be related set of images $\mathrm{I}+$, unrelated set of images IO,

Step three: Start Prepare the sample set of functions SVM algorithm (x i, y i),

Step four: the use of SVM algorithm to calculate the sample build classification surface.

Then added function sign $(\bullet)$,to obtain the maximum distance with the query image.

Step five: for every one picture I $i$, seek score (I i $)=-f(x$ i)

Step Six: according to all the picture score, ascending order and returns the result.

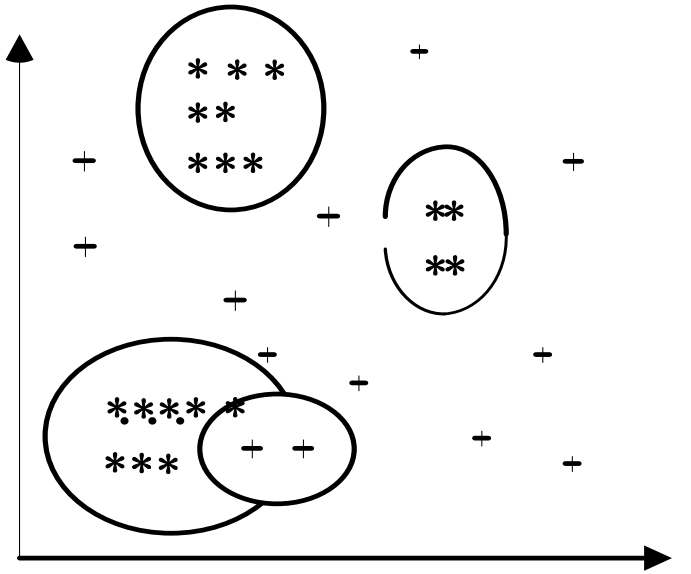

Figure 3 classification

Because users can get the image and the image characteristics similar to retrieve each time, but they can be classified surface [10] distinguish different image information, which can guarantee the retrieval of information is retrieved the next time the user can obtain the desired. It can be seen, with higher precision image retrieval based on SVM relevance feedback image retrieval algorithm retrieval technology.

\section{Retrieval comparison summary}

From the point of view of image retrieval accuracy, color-based image retrieval technology is relatively simple, from a technical level, relatively easy to implement, but the error is relatively large presence, the accuracy is relatively low; knowledge-based image retrieval technology need to be able to identify semantic intelligent system as a support, the system can identify the user relatively accurate information, has higher retrieval accuracy; based on SVM relevance feedback image retrieval retrieval algorithm is based on statistical evolved, the system can automatically retrieve the second, with a high retrieval accuracy.

Three image retrieval technology in applications complement its own advantages, widely used to meet the general user retrieval tasks, but in the face of emerging picture information,there is further room for improvement in retrieval accuracy, which requires researchers on the basis of prior art, painstaking research, the development of image retrieval technology to the next level with higher retrieval accuracy of image retrieval technology and promote.

\section{References}

[1] Xiao-Yu, Li sky. Matching algorithm based on local multi-scale spatial constraints [J]. Modern computer (Professional Edition) 2016 (03) 
[2] Zhou Yi Fei Shumin, Shen Jie. Detect abnormal behavior based real feature point trajectory [J]. Industrial control computer. 2016 (02)

[3] Tao Tao, Zhang Yun. Logarithmic polar coordinate system scale-invariant feature detection and description of points $[\mathrm{J}]$. Chinese Journal of Image and Graphics. 2015 (12)

[4] Feng Wang, Lanfen Lin, Min Tang. A new sketchbased 3D model retrieval approach by using global and local features [J]. Graphical Models. 2013

[5] Guang-Hai Liu, Jing-Yu Yang. Content-based image retrieval using color difference histogram [J]. Pattern Recognition. 2013 (1)

[6] Zhu Jie, Liu Bo, super wooden Japanese power grid, to the sword weighted representation method based on image characteristics of an object's color [J]. Journal of Nanjing University (Natural Science). 2016 (01)

[7] Huang Ningning, CONSERVATORY, Xiao Xuan Hong, Liang Fang Xi, Fan Min. Study Scene image similarity detection algorithm $[\mathrm{J}]$. Science and Technology Outlook. 2015 (34)

[8] $\mathrm{Lu}$ waves multimodal media information retrieval research [D]. Northeastern University, 2013

[9] Qin Fei, Wang Ronggui, Kelvin Leung Hong, Zhang Dongmei, Li Xiang Better TLD-based targets key feature point tracking algorithm [J]. Computer Engineering and Applications. 2016 (04)

[10] Xu Chenghui, Liu Guihua, Liang Feng. Non-feature point Binocular Ranging Technique [J]. ITS Applications. $2013(22)$

[11] Zhao perseverance, Xia Yan. Disordered automatic image matching and recognition [J]. Journal of Computer Applications and Software]. 2014 (12) 